\title{
In-plane optical anisotropy in self-assembled Ge quantum dots induced by interfacial chemical bonds
}

\author{
C. M. Wei, T. T. Chen, and Y. F. Chen ${ }^{\text {a) }}$ \\ Department of Physics, National Taiwan University, Taipei 106, Taiwan, Republic of China \\ Y. H. Peng and C. H. Kuan \\ Department of Electrical Engineering, National Taiwan University, Taipei 106, Taiwan, Republic of China \\ and Graduate Institute of Electronics Engineering, National Taiwan University, Taipei 106, Taiwan, \\ Republic of China
}

(Received 10 October 2006; accepted 8 January 2007; published online 7 February 2007)

\begin{abstract}
In-plane optical anisotropy has been observed in self-assembled Ge quantum dots (QDs). It is found that the photoluminescence (PL) spectrum polarized along [110] exhibits different features compared to that corresponding to [1] 0$]$. Besides, the polarized PL spectrum is able to reveal a detailed fine structure much more pronounced than that in the unpolarized spectrum. It is shown that the observed optical anisotropy is a result of the inherent property of the type-II band alignment of Ge QDs embedded in Si matrix. The light emission arises from the recombination of electrons and holes across the interface, and it thus reflects the anisotropic nature of the interfacial chemical bonds. (C) 2007 American Institute of Physics. [DOI: 10.1063/1.2459506]
\end{abstract}

The physical limitations due to the indirect band gap restrict the luminescence efficiencies in SiGe-based structures. To overcome this difficulty, lots of investigations show that structures based on self-assembled nanostructures such as self-assembled Ge quantum dots (QDs) can enhance the radiative recombination of SiGe-based devices and have a promising application for optical communication in the 1.3-1.5 $\mu \mathrm{m}$ wavelength. ${ }^{1}$ Due to the large band offset and strong quantum confinement in QDs, the optical characteristics can be greatly improved, which has stimulated intensive studies for the development of optoelectronic devices, including growth mechanisms, and electrical and optical properties. ${ }^{2-4}$ However, little has been done on the part of optical anisotropic properties. Optical anisotropy is one of the important characteristics for optoelectronic device applications, such as determining the polarization of lasers and making polarized photodetectors. In this letter, we report an interesting property of interface-induced in-plane optical anisotropy in self-assembled Ge QDs. We demonstrate that the observed optical anisotropy is an intrinsic property of the type-II band alignment of self-assembled Ge QDs embedded in Si matrix. Because the light emission arises from the recombination of electrons and holes across the interface, it reflects the anisotropic characteristics of the interfaces. To further confirm our proposed mechanism, the predicted results for $\mathrm{SiGe} / \mathrm{Si}$ quantum wells and superlattices have been tested, and they are in good agreement with the experimental measurements.

The self-assembled Ge QDs with the fold number of 10 were grown on a $p^{+} \mathrm{Si}(001)$ substrate by a commercial ultrahigh-vacuum chemical vapor deposition system. The Si wafer was cleaned by a dip in HF solution, prior to the deposition of multiple QDs. The growth temperature was maintained at $600{ }^{\circ} \mathrm{C}$ by vacuum annealing furnace. After a $100 \mathrm{~nm}$ thick Si buffer, the ten Ge/Si bilayers were grown including 13.1 equivalent monolayers (1 equivalent monolaers $=6.27 \times 10^{14} \mathrm{Ge}$ atom $/ \mathrm{cm}^{2}$ ) $\mathrm{Ge}$ and $28 \mathrm{~nm} \mathrm{Si}$

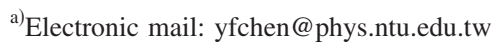

spacer to reduce the roughness of the Ge QD surface. Then, $100 \mathrm{~nm}$ thick undoped Si blocking layer and top Si layer of $100 \mathrm{~nm}$ thickness with boron doping were grown on the multiple QDs. As shown in the inset of Fig. 1, the transmission electron microscopy (TEM) image reveals that the lateral sizes are about $80-140 \mathrm{~nm}$ and the average height is about $6 \mathrm{~nm}$. The PL spectra were recorded by a Spectra Pro 300i monochromator and an InGaAs detector using standard lock-in technique. In order to investigate the optical anisotropy, in front of the entrance slit of the monochromator were an infrared polarizer and a depolarizer which can eliminate the spurious polarization effect arising from the grating. The sample was placed inside a close-cycle He cryostat. A semiconductor laser diode with wavelength of $808 \mathrm{~nm}$ was used as the excitation source.

Figure 1 shows the PL spectrum of the sample at $20 \mathrm{~K}$. The three peaks at $1.142,1.102$, and $1.040 \mathrm{eV}$ are assigned to a transverse-acoustic (TA) phonon, a transverse-optical (TO) phonon, and a TO phonon plus a zone-center-optical phonon $\left(\mathrm{TO}+\mathrm{O}^{\Gamma}\right)$ assisted radiative recombination occurring

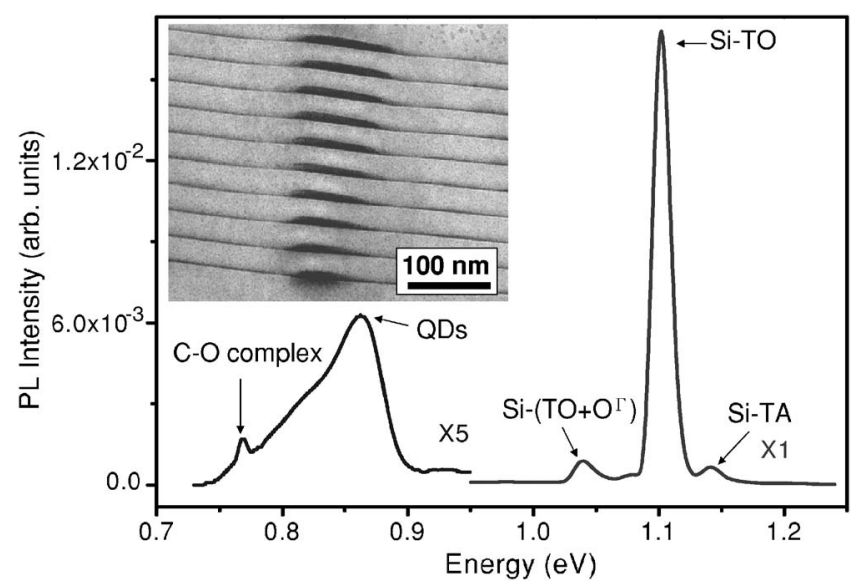

FIG. 1. Photoluminescence spectrum of self-assembled Ge quantum dots under excitation with wavelength of $808 \mathrm{~nm}$. The inset is the cross-sectional transmission electron microscopy image of the sample in this study. 


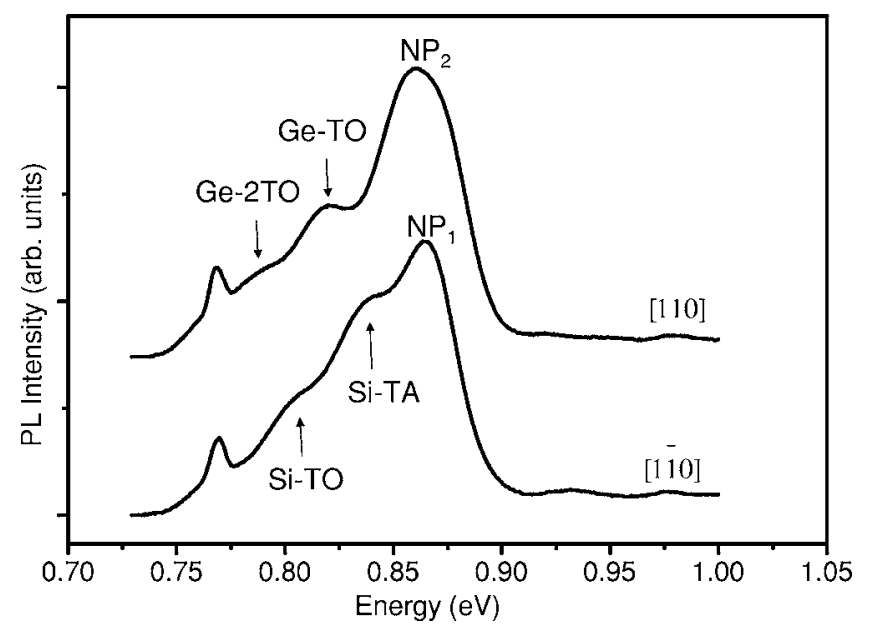

FIG. 2. Polarized photoluminescence spectra of self-assembled Ge quantum dots in the $[1 \overline{10}]$ and $[110]$ directions.

in the $\mathrm{Si}$ substrate, respectively. ${ }^{5,6}$ The sharp peak around $0.768 \mathrm{eV}$ results from $\mathrm{C}-\mathrm{O}$ complex in the Si substrate. ${ }^{6}$ The broad luminescence located around $0.86 \mathrm{eV}$ originates from the recombination of the holes confined in the Ge QDs and the electrons confined in the Si spacers.

An interesting phenomenon is found as we perform the polarization-dependent PL measurement as shown in Fig. 2. For the PL from Ge QDs, the polarization along the [1- 0$]$ and [110] directions are $90^{\circ}$ out of phase, and the corresponding main peaks are denoted as NP1 and NP2, respectively. In contrast, the signals from $\mathrm{Si}$ substrate do not have the same polarization dependence. Thus, the observed optical anisotropy can be attributed to the intrinsic property of $\mathrm{Ge}$ QDs. Previously, optical anisotropy has been attributed to geometrical effects in InAs QDs. ${ }^{7,8}$ However, the scanning electron microscopy and atomic force microscopy images of an uncapped Ge QD sample with similar fabrication processes (not shown here) reveal that the Ge QDs have uniform and symmetrical circular shape. Hence, the optical anisotropy found in our sample cannot be the geometrical effect of Ge QDs.

In order to probe the origin of the optical anisotropy, the measurements of the excitation pumping power and temperature dependences have been performed. It is found that the optical anisotropy is insensitive to the change of excitation pumping power in the range from 10 to $700 \mathrm{~mW}$ and also stable with respect to the change of temperature from 20 to $300 \mathrm{~K}$. These results can be used to rule out the possibility of extrinsic mechanisms responsible for the in-plane anisotropy. For example, the built-in fields caused by unintentional doping will be screened under light irradiation. We can also exclude a significant role of localized states and nonradiative channels in the formation of the in-plane anisotropy, since they will be gradually saturated by the excitation source and the thermally activated carriers will redistribute among them, which will change the transitions of carriers, and hence affect the optical anisotropy. Besides, strain and consequent valence band mixing have also been shown to be important for the optical anisotropy in QDs. ${ }^{9,10}$ Both of them are sensitive to the change of temperature and excitation pumping power. With the change of the excitation pumping power, the electric field across the heterostructure interface will change because of the spatial separation of electrons and Downloaded 04 Mar 2009 to 140.112 .113 .225 . Redistribution subje

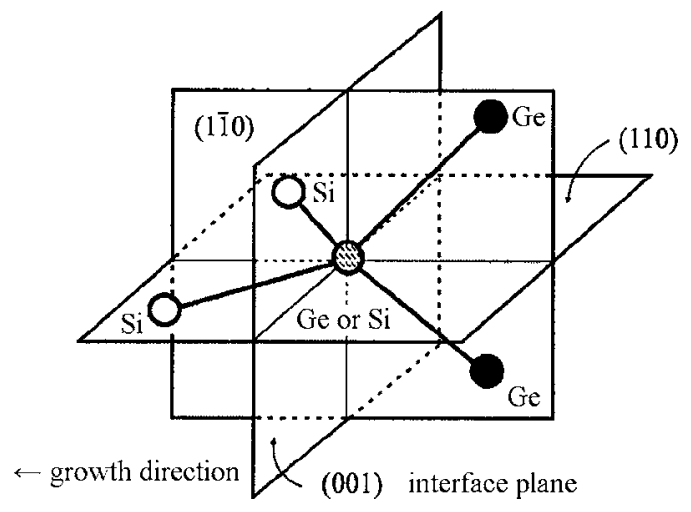

FIG. 3. Schematic diagram of the bond sequence and the polarization of the corresponding interatomic optical transition at the $\mathrm{Si} / \mathrm{Ge}$ interface.

holes. Due to the existence of photoelastic effect in lattice mismatched heterostructures, ${ }^{11}$ the strain and the valence band mixing will also change. However, as mentioned above, it is found that the optical anisotropy is stable with respect to the change of temperature and excitation pumping power. This result therefore provides a solid foundation to rule out the possibility that strain and valence band mixing are important factors in determining our observed optical anisotropy.

To search for the underlying mechanism of the optical anisotropy, let us look at the TEM result. The TEM image shows that the Ge QDs have flat top structures and large lateral size. Due to the large lateral size of Ge QDs, the lateral confinement of holes could be neglected and only the confinement of holes in the growth direction dominates. According to previous reports, ${ }^{12,13}$ the Ge content is much more in the apex region than in the bottom of Ge QDs, which makes the band offset smaller at the bottom of Ge QDs and larger at the top, and consequently the holes would be confined in the apex region. Hence, the radiative recombination mainly occurs at the top interface of Ge QDs.

After realizing the location of the radiative recombination, we now consider the configuration of the chemical bonds across the interface as shown in Fig. 3. Based on the diamond structure of $\mathrm{Si}$ and Ge crystals, the interfacial atom at the top of Ge QDs consists of bonds in a (110) or a $(1 \overline{10})$ plane. It is obvious that these bonds across the interface are not equivalent with respect to the bond directions. Therefore, the in-plane anisotropy inherently exists in the selfassembled Ge QDs embedded in Si matrix. The polarization dependence of the PL spectra thus can result from the anisotropy of interfacial chemical bonds, because the light emission arises from the recombination of electrons and holes across the interface. ${ }^{14,15}$

To further understand the polarized characteristics, we examine the fine structure of the polarized PL spectra. Both $\mathrm{Si}$ and $\mathrm{Ge}$ are materials of indirect band gap so that the phonon assisted processes play important roles in the radiative recombination processes of such materials. Therefore, the multi peak structures in the PL spectra can be attributed to phonon assisted processes. ${ }^{4,16}$ According to the study done by Henini et al, ${ }^{8}$ the energies of the TA mode phonon and the TO mode phonon in $\mathrm{Si}$ are 18.3 and $57.8 \mathrm{meV}$, respectively. In addition, Weber and Alonso's research shows that the phonon energies of Si-TO and $\mathrm{Ge}-\mathrm{TO}$ are 58 and $36 \mathrm{meV}$, respectively. ${ }^{17}$ Therefore, for the polarized PL spectra along to AIP license or copyright; see http://apl.aip.org/apl/copyright.jsp 


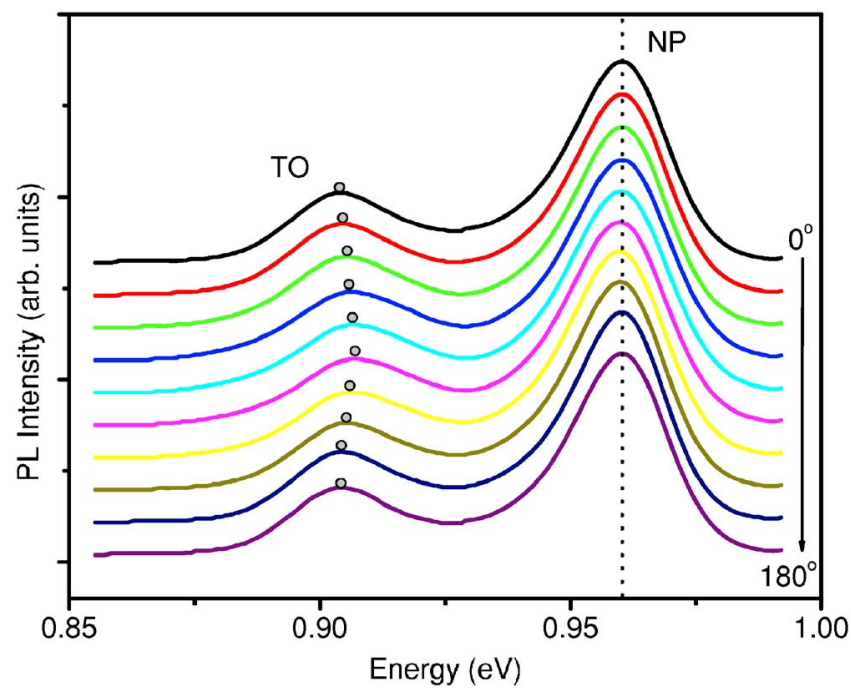

FIG. 4. (Color online) Polarized photoluminescence spectra of $\mathrm{Si}_{0.8} \mathrm{Ge}_{0.2} / \mathrm{Si}$ multiple quantum wells.

the $[1 \overline{1} 0]$ direction, the peaks around 0.84 and $0.8 \mathrm{eV}$ are assigned to a Si-TA phonon assisted transition and a $\mathrm{Si}-\mathrm{TO}$ phonon assisted transition, respectively. For the polarization along the [110] direction, the peaks around 0.820 and $0.785 \mathrm{eV}$ are ascribed to Ge-TO and Ge-2TO phonon replica, respectively. The fact that the phonon replica of $\mathrm{Si}$ and Ge are observed in the PL polarized along the $[1 \overline{1} 0]$ and [110] directions, respectively, can be easily understood according to the chemical bonds across the interface as shown in Fig. 3. For instance, when the emitted light and the polarization are along the [001] and [1 $\overline{1} 0]$ directions, respectively, the interfacial chemical bonds in this geometry are connected with $\mathrm{Si}$ atoms. As a result, $\mathrm{Si}$ related phonon replica dominate. On the other hand, if the polarization is along the [110] direction, the interfacial atom is now connected with Ge atoms, and hence Ge related phonon replica dominates. The fine structure of the phonon replica therefore provides a strong evidence to support our proposed mechanism that the observed optical anisotropy is indeed due to interfacial chemical bonds.

It is worth noting that if the above proposed mechanism is correct, one should expect that the behavior of the in-plane optical anisotropy can also be observed in $\mathrm{Si}_{0.8} \mathrm{Ge}_{0.2} / \mathrm{Si}$ multiple quantum wells (MQWs). Quite interestingly, we found that the optical anisotropy indeed exists in $\mathrm{Si}_{0.8} \mathrm{Ge}_{0.2} / \mathrm{Si}$ MQWs as shown in Fig. 4. The no phonon (NP) assisted transition is isotropic, while the TO phonon replica is not. Because the NP transition arises from the relaxation of momentum selection rule due to alloy potential fluctuations, it does not reflect the structure anisotropy. The variation of the TO phonon replica can be explained in terms of the anisotropy of interfacial chemical bonds as described above, in which TO phonon has a different energy in different orientations. This observation is thus consistent with the prediction according to our proposed model.
We have also performed the same experiment for $\mathrm{Si}_{0.5} \mathrm{Ge}_{0.5} / \mathrm{Si}$ superlattices (SLs) without Ge QDs and found no indication of optical anisotropy. It can be interpreted in terms of the fact that the quantum tunneling occurs for the electrons and holes in $\mathrm{Si}_{0.5} \mathrm{Ge}_{0.5} / \mathrm{Si}$ SLs and the minibands are formed. Under this circumstance, the radiative recombination process at the interface between $\mathrm{SiGe}$ and $\mathrm{Si}$ does not dominate the light emission, and the optical anisotropy induced by interfacial chemical bonds disappears. This behavior again provides a further evidence to support our proposed mechanism.

In summary, optical anisotropy has been observed in self-assembled Ge QDs. We show that the anisotropy arises from the inherent nature of the type-II band alignment $\mathrm{Ge}$ QDs embedded in Si matrix. Because the transition is now due to the recombination of electrons and holes across the interface, the light emission reflects the anisotropic characteristic of the interfacial chemical bonds. The predicted results according to our proposed mechanism have also been verified for the cases of SiGe/Si MQWs as well as SLs. We therefore firmly establish an intriguing optical behavior in $\mathrm{Si} / \mathrm{Ge}$ heterostructures due to their type-II band alignment, which should be very useful for their application in optoelectronic devices.

This research was supported by the National Science Council and Ministry of Education of the Republic of China.

${ }^{1}$ J. C. Campbell, in Germanium Silicon: Physics and Materials: Optoelectronics in Silicon and Germanium Silicon, edited by R. Hull and J. C. Bean (Academic, San Diego, 1999), 56, p. 347.

${ }^{2}$ T. Brunhes, P. Boucaud, S. Sauvage, F. Aniel, J.-M. Lourtioz, C. Hernandez, Y. Campidelli, O. Kermarrec, D. Bensahel, G. Faini, and I. Sagnes, Appl. Phys. Lett. 77, 1822 (2000).

${ }^{3}$ O. G. Schmidt and K. Eberl, Phys. Rev. B 61, 13721 (2000).

${ }^{4}$ V. Le Thanh, V. Yam, P. Boucaud, F. Fortuna, C. Ulysse, D. Bouchier, L. Vervoort, and J.-M. Lourtioz, Phys. Rev. B 60, 5851 (1999).

${ }^{5}$ P. J. Dean, J. R. Haynes, and W. F. Flood, Phys. Rev. 161, 711 (1967).

${ }^{6}$ W.-H. Chang, W.-Y. Chen, A.-T. Chou, T.-M. Hsu, P.-S. Chen, Z. Pei, and L.-S. Lai, J. Appl. Phys. 93, 4999 (2003).

${ }^{7}$ H. Saito, K. Nishi, S. Sugou, and Y. Sugimoto, Appl. Phys. Lett. 71, 590 (1997).

${ }^{8}$ M. Henini, S. Sanguinetti, S. C. Fortina, E. Grilli, M. Guzzi, G. Panzarini, L. C. Andreani, M. D. Upward, P. Moriarty, P. H. Beton, and L. Eaves, Phys. Rev. B 57, R6815 (1998).

${ }^{9}$ W. Yang, H. Lee, T. J. Johnson, P. C. Sercel, and A. G. Norman, Phys. Rev. B 61, 2784 (2000).

${ }^{10}$ S. Cortez, O. Krebs, P. Voisin, and J. M. Gérard, Phys. Rev. B 63, 233306 (2001).

${ }^{11}$ C. H. Chen, W. H. Chen, Y. F. Chen, and T. Y. Lin, Appl. Phys. Lett. 83, $1770(2003)$

${ }^{12}$ C. Lang, D. J. H. Cockayne, and D. Nguyen-Manh, Phys. Rev. B 72, 155328 (2005).

${ }^{13}$ J.-M. Baribeau, X. Wu, and D. J. Lockwood, J. Vac. Sci. Technol. A 24, 663 (2006).

${ }^{14}$ A. V. Platonov, V. P. Kochereshko, E. L. Ivchenko, G. V. Mikhailov, D. R. Yakovlev, M. Keim, W. Osau, A. Waag, and G. Landwehr, Phys. Rev. Lett. 83, 3546 (1999).

${ }^{15}$ W. S. Su, M. H. Ya, Y. S. Chiu, and Y. F. Chen, Phys. Rev. B 66, 113305 (2002).

${ }^{16}$ J. Wan, G. L. Jin, Z. M. Jiang, Y. H. Luo, J. L. Liu, and Kang L. Wang, Appl. Phys. Lett. 78, 1763 (2001).

${ }^{17}$ J. Weber and M. I. Alonso, Phys. Rev. B 40, 5683 (1989). 